\title{
ANÁLISIS DEL ÁREA SOCIOFAMILIAR Y LABORAL EN LAS PERSONAS AFECTADAS POR EL SÍNDROME DE ARDYSTIL
}

\section{ANALYSIS OF THE SOCIO-FAMILIAL AND LABOR AREA IN THE PEOPLE AFFECTED BY THE SYNDROME OF ARDYSTIL}

Recibido/Received: 19/05/2010

Aceptado/Accepted: 18/09/2010

\section{RESUMEN}

Se realiza una revisión teórica de la Psicología de las Organizaciones, desde los inicios históricos de la Psicología del Trabajo, analizando las distintas teorías, hasta llegar al concepto de Organización. Las estadísticas sobre siniestralidad laboral sitúan a España como uno de los países donde se producen más accidentes laborales en el ámbito de la Unión Europea. El artículo 40.2 de la Constitución Española encomienda a los poderes públicos, como uno de los principios rectores de la política social y económica, velar por la seguridad e higiene en el trabajo. Al cumplimiento de este mandato responde la Ley de Prevención de Riesgos Laborales 31/1995 de 8 de noviembre. En este estudio empírico se pretenden valorar aspectos relacionados con la vida diaria del sujeto y en qué modo se ve perjudicado éste por la presencia de la patología en el área sociofamiliar y laboral, después de producirse en 1992 un acontecimiento tan grave para la salud, debido a la exposición de trabajadores del sector textil a la enfermedad denominada como "Síndrome Ardystil", que provocó muertes y graves secuelas. Este trabajo analiza dichas secuelas entre los antiguos trabajadores.

\section{PALABRAS CLAVE}

Accidentes laborales, cultura preventiva, salud laboral, prevención de riesgos laborales, psicosociología aplicada.

\section{SUMARIO}

1. Introducción. 2. Objetivos. 3. Métodos. 4. Resultados. 5. Conclusiones y discusión. Bibliografía.

\begin{abstract}
At first there is done a theoretical review of the Psychology of the Organizations, analyzing it from the historical beginnings of the Psychology of the work, through different theories, up to coming to the concept of Organization. The statistics on accidents at work place Spain as one of the countries where there are more accidents in the area of the European Union. The article 40.2 of the Constitution assigns to the government, as one of the guiding principles of the social and economic policy, to ensure the health and safety at work. To the fulfilment of this mandate responds the Law
\end{abstract}


of Prevention of Labour Risks 31/1995, November $8^{\text {th }}$. For this empirical study there try to be valued aspects related to the daily life of the subject and for what way this one sees harmed by the presence of the pathology in the social family and labour area, after such a serious event took place for the health, due to exposure of textile workers to the disease known as "Syndrome of Ardystil", which caused deaths and serious injuries. This work analyzes the above mentioned sequels among the former workers.

\section{KEYWORDS}

Work accidents, preventive culture, occupational health, accident-prevention, applied social psychology.

\section{CONTENTS}

1. Introduction. 2. Objectives. 3. Methods. 4. Results. 5. Conclusions and discussion. References.

\section{INTRODUCCIÓN}

En la sociedad contemporánea, en su evolución histórica, el concepto, el número de organizaciones, y su importancia, han sido elemento fundamental para su desarrollo. Según Peiró (1996), han intervenido numerosos factores como son los técnicos, legales, estructurales e individuales.

El funcionamiento de la organización se va a concebir como un sistema mecánico cerrado y como un sistema burocrático-despersonalizado; consideraciones que surgen, respectivamente, desde la ingeniería, con la teoría de la organización científica de Taylor y con la teoría de la administración científica de Fayol (Rodríguez et al., 2004) y desde la Sociología con la aportación de Weber y su teoría de la organización burocrática. La teoría de la burocracia de Weber, es la primera que ofrece una visión sistemática sobre las organizaciones. Ofrece una visión estructural de la organización y aborda su estudio desde un nivel global (Osca, 2004).

Como señala Palací (2005), en un primer momento las limitaciones de la concepción mecanicista se representan en los estudios sobre el "factor humano". Dichos estudios suponen una contribución relevante en el origen y desarrollo de la Psicología Industrial.

Se produce una superación crítica de la posición individualista, se considera la importancia del contacto y la interacción del sujeto con otros de la misma organización. En este contexto surge la "Escuela de Relaciones Humanas" que va a constituir el primer esfuerzo de investigación empírica en la industria y la primera conceptualización de la organización sustentada teóricamente (Ibarra, 2000). Su inicio hay que situarlo en el trabajo de Elton Mayo [Mayo, (1933) y Roethlisberger, y Dickson, (1939)] y sus experimentos en la Western Electric Company.

En el último conjunto de aportaciones vinculadas a la Escuela de Relaciones Humanas, que convirtió el liderazgo en uno de los principales temas de interés, las conexiones entre la investigación industrial y la Psicología Social se hicieron más que obvias.

El estilo de liderazgo era importante para predecir el nivel de rendimiento de los trabajadores, por lo que se preconizaba la implantación de un estilo de dirección 
democrático y participativo para incrementar la moral de los empleados y, con ello, la productividad de las empresas, según Martín-Quirós y Zarco (2009).

Surgirá un modelo denominado "sociotécnico", consistente en incorporar las dimensiones técnicas, humanas y sociales, para explicar el comportamiento laboral en la organización.

Por otro lado, están los "modelos humanistas" que derivan de la corriente de la psicología humanista y parten de una concepción del individuo que contradice y supera las suposiciones tradicionales, a la vez que sirve de complemento a las proposiciones realizadas por la escuela de relaciones humanas (Rodríguez et al., 2004; Martín-Quirós y Zarco, 2009). Consideran a todos los miembros de la organización como fuentes de recursos, se incluyen no sólo las habilidades físicas, sino también la capacidad creativa y la presencia de conductas responsables, autodirigidas y autocontroladas.

Se presenta a un individuo autodisciplinado, orientado hacia el logro personal y el crecimiento psicológico. Uno de los autores más representativos es Maslow (1943) que desarrolló una jerarquía de necesidades que ha tenido una amplia repercusión en la teoría y práctica organizacional.

La "teoría del equilibrio" propugnada por Simon y colaboradores pretende establecer un modelo de carácter integrado con respecto a los modelos anteriores. Esta perspectiva integradora se puede realizar tomando como referencia la "teoría general de sistemas", cuya pretensión era la de crear una ciencia universal que utilizara los elementos organizacionales comunes, que se encuentran en todos los sistemas.

Las organizaciones son realidades sociales en continuo desarrollo y cambio, lo que implica el estudio de dichos cambios y la necesidad de tener en consideración la constante evolución histórica en las instituciones. El fenómeno organizacional es una realidad muy frecuente en todo país desarrollado, de forma que se ha llegado a afirmar que cuanto más desarrollado es un país, más crecen sus organizaciones.

Hay que tener en cuenta que las necesidades y objetivos de los individuos y de las organizaciones están constantemente cambiando, el mantenimiento del "ajuste entre la persona y el entorno" requiere esfuerzos continuados por parte del individuo y de la organización.

Las organizaciones no sólo están compuestas de individuos, sino que también están integradas por grupos ya sean formales o informales en función de su origen y de las funciones que cumplan.

Las organizaciones se definen como sistemas sociales, relativamente permanentes, constituidos por individuos y grupos de personas con unos objetivos o metas que tratan de lograr, dotándose de una estructuras y de una cultura que les permita llevar a cabo los procesos pertinentes de modo eficaz y satisfactorio para todos sus miembros, así como para el entorno en el que se ubica.

Al mismo tiempo que se ha ido avanzando en la evolución productiva, se ha ido produciendo una constante progresión en la regulación de la seguridad y salud de los trabajadores, teniendo como pieza central la extinguida Ordenanza General de Seguridad e Higiene en el Trabajo, aprobada por la Orden de 9 de marzo de 1971.

Se ha realizado un importante esfuerzo de promulgación de normas tendentes a confeccionar un marco jurídico de la prevención lo más completo posible hasta llegar a la promulgación de la Ley de Prevención de Riesgos Laborales, que ha supuesto un cambio 
radical en el Derecho de la Seguridad y Salud Laboral en nuestro país y se ha convertido en uno de los más importantes centros de imputación jurídica del ordenamiento laboral.

La Ley 31/1995, de 8 de noviembre, pretende dotar de los instrumentos jurídicos necesarios para conseguir la mejora de las condiciones de trabajo y elevar la protección de la salud y seguridad de los trabajadores. La Ley abandona deliberadamente una concepción reparadora y proteccionista y se sitúa en una fase previa a la materialización de los daños. De este modo, se actúa sobre las condiciones de trabajo para evitar que los riesgos se actualicen y, con ellos, se produzcan daños en la salud de los trabajadores (Romeral, 2007).

El concepto de condiciones de trabajo, central en la Ley, no se reduce a las condiciones físicas o ambientales de los lugares de trabajo. Las condiciones organizacionales y psicosociales son factores reales de riesgo, como lo son los factores físicos y ambientales (Moreno, 2000).

Esta investigación se centra en la comarca Alcoiá-Comtat en la Comunidad Valenciana, donde la industria principal es la fabricación de toda clase de textiles, confección y estampación.

En los años 90 hubo una fortísima recesión económica-industrial, siendo las consecuencias fácilmente imaginables:

- Aumento del desempleo

- Precariedad en el empleo

- Economía sumergida creciente

Dentro de este contexto aparece la actividad del aerografiado textil, caracterizado por ser un proceso básicamente manual y no-mecanizado, una nueva implantación dentro del sector textil, que se encuentra oficialmente englobada dentro de una actividad industrial denominada acabados textiles.

La aerografía es un procedimiento empleado en las artes gráficas, que permite obtener dibujos atrayentes con colores de efectos sensualistas. La técnica consiste en pulverizar una pintura mediante una pistola de aire, que valiéndose del efecto Venturi, la proyecta atomizada sobre la tela delimitándose los dibujos mediante unas plantillas.

Este sistema, al pulverizar pinturas que se consiguen por la mezcla de productos químicos, crea una atmósfera agresiva que agrava su efecto, creando un microclima que afecta al organismo, cuando los espacios de trabajo son reducidos y carecen de una ventilación adecuada.

Este sencillo sistema permite construir una instalación industrial con una inversión relativamente reducida, lo que facilita la generación de empresas muy pequeñas y sin personal técnico cualificado.

Las características de estas empresas son:

- Empresas muy pequeñas, en cuanto a las dimensiones de las mismas.

- Número de trabajadores, y medidas de protección para la salud muy escasas o nulas.

- Desconocimiento por parte de los trabajadores de la peligrosidad de los productos que se utilizan, jornadas y condiciones de trabajo.

En algunos trabajadores que se dedicaban al aerografiado empiezan a aparecer síntomas físicos, como mareos, tos fuerte, hemorragias nasales. Durante el año 1992 fallecen seis personas, y hay un total de setenta personas afectadas, las cuales hoy en día aún continúan pasando reconocimientos médicos para controlar la enfermedad. 


\section{OBJETIVOS}

Los accidentes de trabajo se han convertido en uno de los fenómenos más disfuncionales del mundo laboral, por sus consecuencias, su constante aumento y sus dificultades para poder establecer programas de prevención eficaces y duraderos. Dentro de esta temática, y respecto al síndrome Ardystil, se plantea los siguientes objetivos:

- Estudiar la evolución de la variable "calidad de vida" en el área sociofamiliar y laboral después de producirse un acontecimiento tan grave para la salud como el síndrome Ardystil.

- Verificar la evolución de la calidad de vida en los sujetos afectados por el Síndrome Ardystil en el área sociofamiliar y laboral.

\section{MÉTODO}

\section{SUJETOS}

Tabla 1. Sexo

\begin{tabular}{|l|c|c|c|c|}
\hline \multirow{2}{*}{ Válidos } & & Frecuencia & Porcentaje & $\begin{array}{c}\text { Porcentaje } \\
\text { acumulado }\end{array}$ \\
\cline { 2 - 6 } & mujer & 60 & 69,8 & 69,8 \\
\cline { 2 - 6 } & hombre & 26 & 30,2 & 100,0 \\
\hline \multirow{2}{*}{ Perdidos } & Total & 86 & 100,0 & \\
\hline \multicolumn{2}{|c|}{ Sistema } & 0 & & \\
\hline
\end{tabular}

La muestra está compuesta por 86 sujetos, 45 afectados por el Síndrome Ardystil, y 41 no afectados, constituyendo estos últimos la muestra de control. De todos ellos, 60 son mujeres y 26 hombres, cuyas edades oscilan desde los 21 años, la edad mínima, hasta los 59 siendo está la edad máxima. Se distribuyen mayoritariamente desde los 21 con una frecuencia de 2 hasta los 28 con una frecuencia de 10, siendo la edad con más frecuencia los 23 años. Las edades siguientes van desde los 30 años con una frecuencia de 4 hasta los $31,32,33,34,41,43,56$ y 59 con unas frecuencias de 2 sujetos por edad. Obteniendo así una medía de edad de entre 27 y 28 años.

\section{VARIABLES}

En este estudio se ha considerado la existencia de una variable independiente o criterio, consistente en el padecimiento de enfermedad debido al Síndrome Ardystil. Dicha variable tiene dos valores; presencia de enfermedad debido al Síndrome (sujetos enfermos), y grupo de control (sujetos sanos).Las variables dependientes que se ha incluido en el estudio y que se ha considerado que sería de mayor interés para los objetivos propuestos, se enumeran a continuación, todas ellas del área sociofamiliar y laboral:

- Relaciones familiares.

- Apoyo familiar percibido. 
- Satisfacción global con la situación familiar.

- Relaciones sociales extrafamiliares.

- Satisfacción con las relaciones sociales extrafamiliares.

- Situación laboral: satisfacción/mantenimiento del empleo/normalidad en el desempeño del puesto de trabajo/expectativas laborales/jubilación: vivencia de la situación

\section{TÉCNICAS}

El presente trabajo fue abordado en distintas fases. Una vez administrado a la muestra un instrumento de evaluación (consistente en un cuestionario), los datos obtenidos fueron sometidos a un tratamiento estadístico en función del objetivo que se había planteado. Se analizó la información mediante el programa SPSS-16. Se calcularon los estadísticos descriptivos definitorios de cada variable dependiente, y se realizó un análisis de varianza no paramétrico de carácter nominal mediante la técnica de Chi Cuadrado, para establecer la existencia de diferencias entre los dos niveles de la variable independiente. Como prueba post-hoc se realizaron contrastes con el coeficiente de contingencia "C".

Se pretende recabar información sobre la opinión de los sujetos respecto a cómo han cambiado sus capacidades funcionales antes de padecer la enfermedad con respecto al momento actual. Este estudio posee el valor de que dado que ha pasado un tiempo de 10 años como mínimo, la información obtenida permite recoger información que no suele estar presente en otros estudios.

El diseño es correlacional, univariado-multivariado, de tipo cuasiexperimental, con grupo de control.

\section{RESULTADOS}

Los resultados del estudio realizado a los sujetos afectados por el "Síndrome de Ardystil" comparándolo con las personas no afectadas por este síndrome, se muestran en las tablas siguientes, en las que se señala la respuesta más utilizada por cada grupo de sujetos, y el porcentaje de utilización de la misma. Es preciso destacar que en algunas de las preguntas sólo se especifica la información del grupo experimental, ya que la pregunta efectuada al grupo control no tiene sentido, al tratarse de sujetos sanos.

\section{ÁREA SOCIOFAMILIAR}

1. Con respecto a la variable "¿Está usted satisfecho de la reacción de su familia al conocer su enfermedad?", de los 44 sujetos afectados sondeados, un 68,2\% de afirma que está "mucho" o muy satisfecho de la reacción de su familia al conocer su enfermedad. Un $22,7 \%$ de los sujetos contestó que estaba "bastante" satisfecho de la reacción de sus familias al conocer su enfermedad, un 4,5\% respondió estar satisfecho de forma "regular" a la reacción de sus familias, y un 4,5\% indicó no sentirse "nada" satisfecho ante la reacción de sus familias al conocer su enfermedad, como se muestra en la Tabla 1 y en el Gráfico 1.

El estadístico Chi-Cuadrado para la variable ofrece un valor de 47,636 con 3 grados de libertad y una significación asintótica de, 000 . Lo que indica que la relación entre las dos variables es significativa y de dependencia. 
Tabla 2. ¿Está usted satisfecho de la reacción de su familia al conocer su enfermedad?

\begin{tabular}{|l|l|c|c|c|}
\hline & & Frecuencia & Porcentaje & Porcentaje acumulado \\
\hline Válidos & nada & 2 & 4,5 & 4,5 \\
\cline { 2 - 5 } & regular & 2 & 4,5 & 9,1 \\
\cline { 2 - 5 } & bastante & 10 & 22,7 & 31,8 \\
\cline { 2 - 5 } & mucho & $\mathbf{3 0}$ & $\mathbf{6 8 , 2}$ & $\mathbf{1 0 0 , 0}$ \\
\cline { 2 - 5 } & Total & 44 & 100,0 & \\
\hline Perdidos & Sistema & 42 & & \\
\hline Total & & 86 & & \\
\hline
\end{tabular}

Gráfico 1. ¿Está usted satisfecho de la reacción de su familia al conocer su enfermedad?

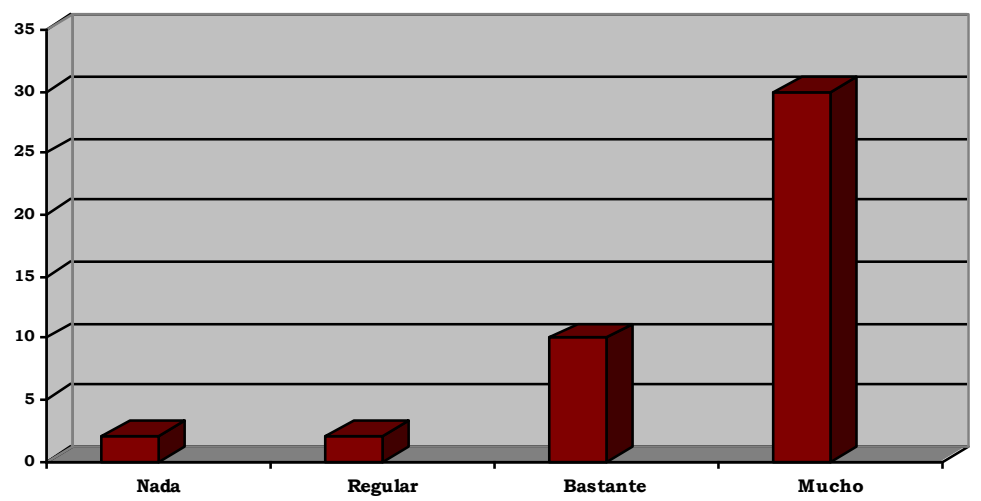

Tabla 3. Prueba de Chi-Cuadrado. ¿Está usted satisfecho de la reacción de su familia al conocer su enfermedad?

\begin{tabular}{|c|c|}
\hline & $\begin{array}{c}\text { ¿Esta usted satisfecho de la reacción de su familia al conocer su } \\
\text { enfermedad? }\end{array}$ \\
\hline Chi-cuadrado & $\mathbf{4 7 , 6 3 6}$ \\
\hline gl & 3 \\
\hline Sig. asintót. &, 000 \\
\hline
\end{tabular}

2. La variable “¿Está usted satisfecho de la reacción de esas personas al conocer su enfermedad?" obtiene una serie de datos obtenidos que han sido representados en la Tabla 4. De los 45 sujetos encuestados, un $40 \%$ de ellos está bastante satisfecho de la reacción de sus amigos/compañeros al conocer su enfermedad, es decir, un total de 18 sujetos de los 45 que contestaron a estar pregunta, como se muestra en el Gráfico de frecuencias. Un 35,6\% de los sujetos contestó que "mucho" o muy satisfechos de la reacción de esas personas al 
conocer su enfermedad, frente a un $8,9 \%$, un total de 4 encuestados, que indicaron que están "regular" y "poco" satisfechos con las reacciones ante su enfermedad, y solamente un $6,7 \%$ contestaron que no estaban nada satisfechos de la reacción de esas personas al conocer su enfermedad.

Gráfico 2. ¿Está usted satisfecho de la reacción de esas personas al conocer su enfermedad?

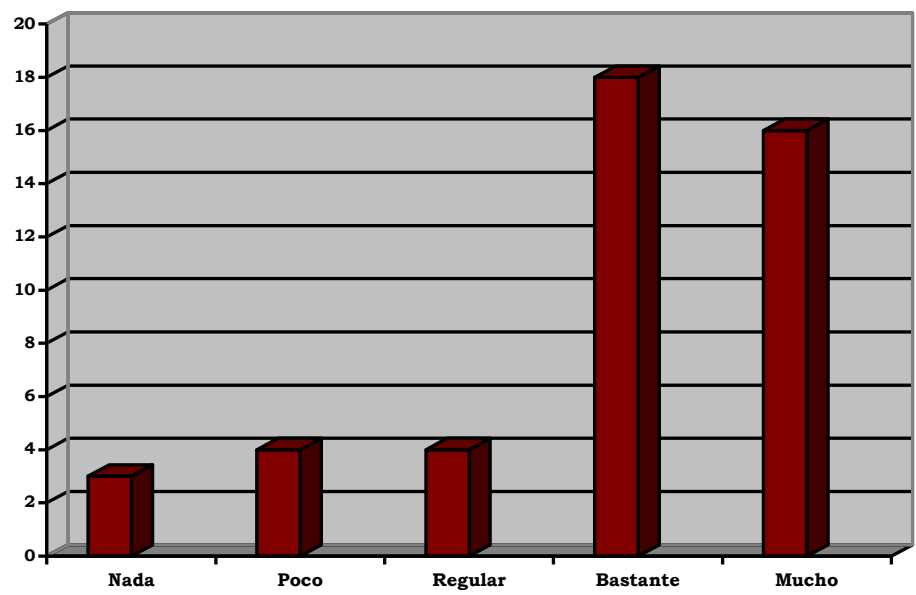

Tabla 4. ¿Está usted satisfecho de la reacción de esas personas al conocer su enfermedad?

\begin{tabular}{|l|l|c|c|c|}
\hline & & Frecuencia & Porcentaje & Porcentaje acumulado \\
\hline \multirow{5}{*}{ Válidos } & nada & 3 & 6,7 & 6,7 \\
\cline { 2 - 5 } & poco & 4 & 8,9 & 15,6 \\
\cline { 2 - 5 } & regular & 4 & 8,9 & 24,4 \\
\cline { 2 - 5 } & bastante & $\mathbf{1 8}$ & $\mathbf{4 0 , 0}$ & $\mathbf{6 4 , 4}$ \\
\cline { 2 - 5 } & mucho & 16 & 35,6 & 100,0 \\
\cline { 2 - 5 } & Total & 45 & 100,0 & \\
\hline Perdidos & Sistema & 41 & & \\
\hline Total & & 86 & & \\
\hline
\end{tabular}

La prueba de Chi-Cuadrado referente a la variable que nos ocupa “¿Está usted satisfecho de la reacción de su familia al conocer su enfermedad?", ofrece un valor de 47,636 con 3 grados de libertad y con una significación asintótica de ,000, lo que indica que existe una relación significativa entre las variables, es decir, la variable “¿Está satisfecho de la reacción de su familia al conocer su enfermedad?" tiene relación de dependencia con la variable "Estar enfermo"; como se observa en la Tabla 3. 
Gráfico 3. En relación con la época anterior a su enfermedad, sus relaciones familiares...?

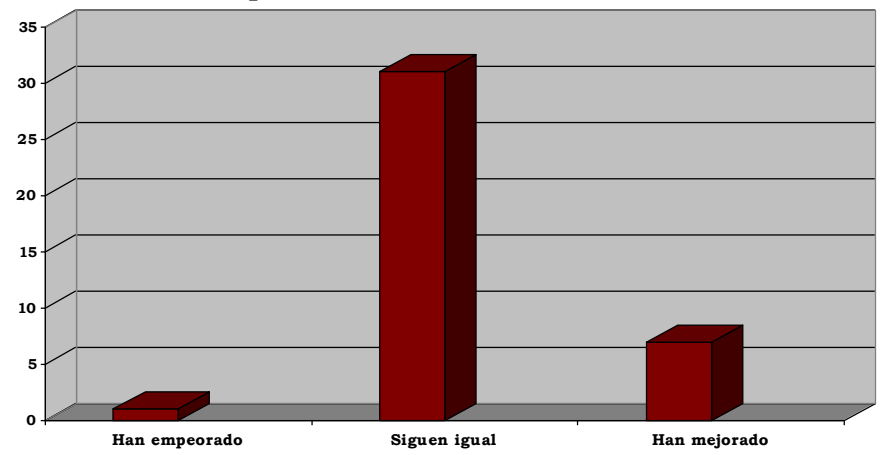

Tabla 4b. Prueba de Chi-Cuadrado. ¿Está usted satisfecho de la reacción de esas personas al conocer su enfermedad?

\begin{tabular}{|c|c|}
\hline & $\begin{array}{c}\text { ¿Esta usted satisfecho de la reacción de su familia al } \\
\text { conocer su enfermedad? }\end{array}$ \\
\hline Chi-cuadrado & $\mathbf{4 7 , 6 3 6}$ \\
\hline gl & 3 \\
\hline Sig. asintót. &, 000 \\
\hline
\end{tabular}

Gráfico 4. En general, su satisfacción con las relaciones que mantiene con las personas que le rodean, después detección de la enfermedad

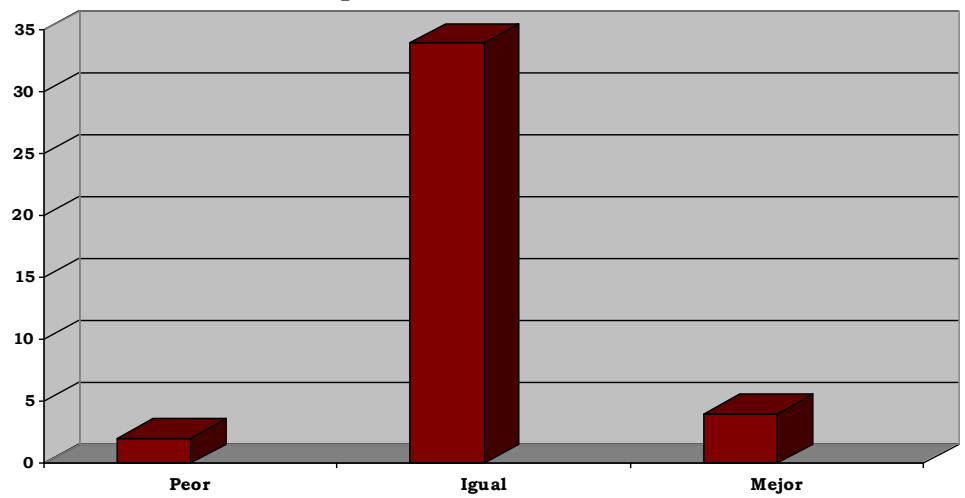

3. La variable "En relación con la época anterior a su enfermedad, sus relaciones familiares..." muestra resultados que se pueden observar en la Tabla 5 de frecuencias y porcentajes. Y que muestra que de los 39 sujetos encuestados, un 79,5\% de ellos afirma que sus relaciones familiares siguen igual, es decir, un total de 31 personas; el 17,9\% dice 
que ha mejorado; y 2,6\% explica que sus relaciones familiares han empeorado, como se observa en el Gráfico 3.

Tabla 5. En relación con la época anterior a su enfermedad, sus relaciones familiares...?

\begin{tabular}{|l|l|c|c|c|}
\hline & & Frecuencia & Porcentaje & Porcentaje acumulado \\
\hline Válidos & han empeorado & 1 & 2,6 & 2,6 \\
\cline { 2 - 5 } & siguen igual & $\mathbf{3 1}$ & $\mathbf{7 9 , 5}$ & $\mathbf{8 2 , 1}$ \\
\cline { 2 - 5 } & han mejorado & 7 & 17,9 & 100,0 \\
\cline { 2 - 5 } & Total & 39 & 100,0 & \\
\hline Perdidos & Sistema & 47 & & \\
\hline Total & & 86 & & \\
\hline
\end{tabular}

Gráfico 5. En relación con la época anterior a la enfermedad sus relaciones con sus amigos, compañeros, etc. son

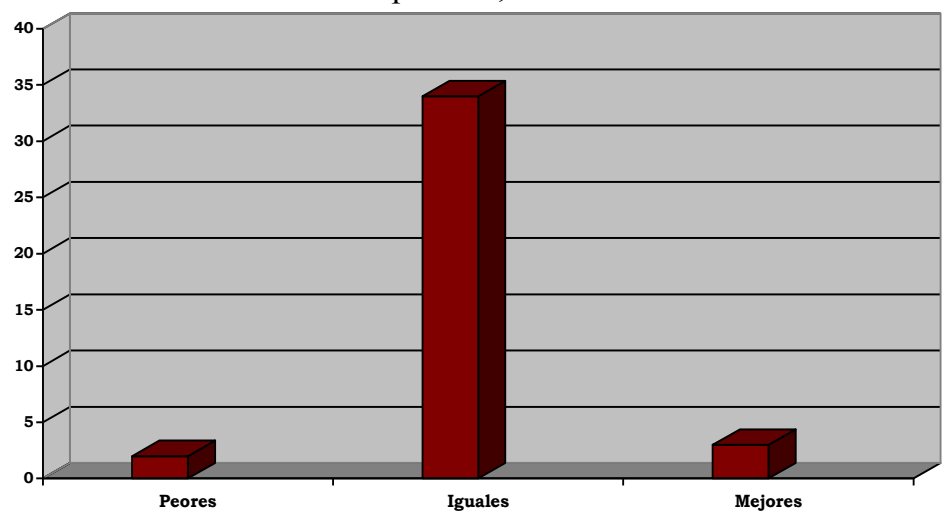

La prueba de Chi-Cuadrado, Tabla 6, realizada para esta variable muestra un valor de 38,769 con 2 grados de libertad y una significación asintótica de ,000. Por lo que se determina que la relación entre las dos variables es significativa, es decir, la variable estar enfermo tiene relación con la variable sobre la relación con sus familiares en la época anterior a su enfermedad.

Tabla 6. Prueba de Chi-Cuadrado. En relación con la época anterior a su enfermedad, sus relaciones familiares:

\begin{tabular}{|c|c|}
\hline & $\begin{array}{c}\text { En relación con la época anterior a su } \\
\text { enfermedad, sus relaciones familiares }\end{array}$ \\
\hline Chi-cuadrado & $\mathbf{3 8 , 7 6 9}$ \\
\hline gl & 2 \\
Sig. asintót. &, 000 \\
\hline
\end{tabular}


4. La variable "En general, su satisfacción con las relaciones que mantiene con las personas que le rodean, después de la detección de la enfermedad..." muestra sus resultados en la Tabla 7 de frecuencias y porcentajes. Indica que de los 45 sujetos afectados por el Síndrome de Ardystil, respondieron a esta pregunta un total de 40, de los cuales un $85 \%$ manifiesta que su satisfacción con las relaciones que mantienen con las personas que les rodean, después de la detección de la enfermedad, es "igual"; el 10\% de los sujetos declara que la relación con las demás personas es "mejor"; y el 5\% de lo afectados manifiesta que su relación es "peor" después de la detección de la enfermedad (un total de 2 personas), como se refleja en el Gráfico 4.

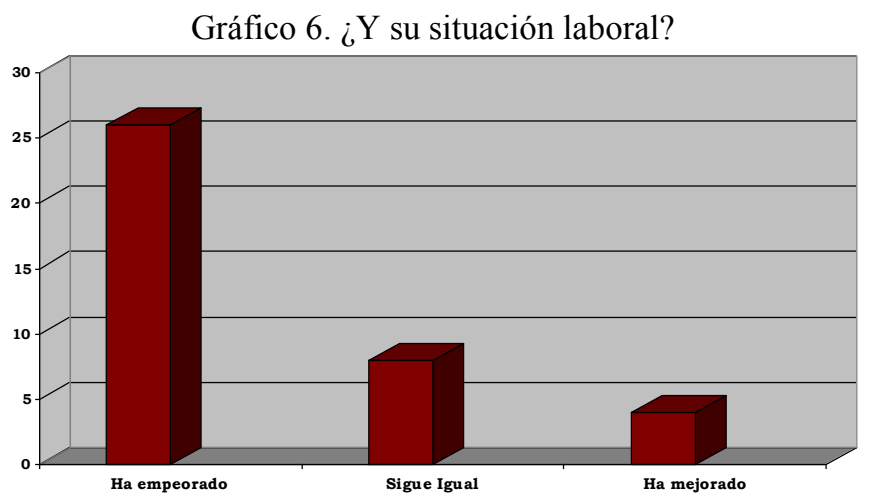

Tabla 7. En general, su satisfacción con las relaciones que mantiene con las personas que le rodean, después detección de la enfermedad

\begin{tabular}{|l|l|c|c|c|}
\hline \multirow{3}{*}{ Válidos } & peor & Frecuencia & Porcentaje & $\begin{array}{c}\text { Porcentaje } \\
\text { acumulado }\end{array}$ \\
\cline { 2 - 5 } & igual & $\mathbf{3 4}$ & 5,0 & 5,0 \\
\hline & mejor & 4 & 10,0 & 100,0 \\
\cline { 2 - 5 } & Total & 40 & 100,0 & \\
\hline Perdidos & Sistema & 46 & & \\
\hline Total & & 86 & & \\
\hline
\end{tabular}

La prueba de Chi-Cuadrado realizada a la variable "En general, su satisfacción con las relaciones que mantienen con las personas que le rodean, después la detección de la enfermedad" ofrece una significación asintótica ,000 cuyo valor es de 48,200 con 2 grados de libertad, lo que indica que la relación entre las variables es significativa, es decir, la 
variable satisfacción con las relaciones que mantiene con las personas que le rodean tiene relación de dependencia con la variable de estar o no afectado por el Síndrome de Ardystil.

Tabla 8. Prueba de Chi-Cuadrado. En general, su satisfacción con las relaciones que mantiene con las personas que le rodean, después detección de la enfermedad.

\begin{tabular}{|c|c|}
\hline & $\begin{array}{c}\text { En general, su satisfacción con las relaciones que } \\
\text { mantiene con las personas que le rodean, después } \\
\text { detección de la enfermedad }\end{array}$ \\
\hline Chi-cuadrado & $\mathbf{4 8 , 2 0 0}$ \\
\hline gl & 2 \\
Sig. asintót. &, 000 \\
\hline
\end{tabular}

\section{ÁREA SOCIOLABORAL}

5. En relación a la evaluación del área sociolaboral, se preguntó a los sujetos del estudio si "En relación con la época anterior a la enfermedad, sus relaciones con sus amigos, compañeros, etc. son...". De los 45 sujetos afectados encuestados, respondieron 39. De ellos, el $87,2 \%$ de los sujetos manifiesta que las relaciones con sus amigos son "iguales" a la época anterior a la enfermedad; el 7,7\% de los sujetos declara que dichas relaciones son "mejores". Y un 5,1\% de los sujetos indica que las relaciones con sus amigos, compañeros, etc., comparadas con la época anterior a la enfermedad, son "peores", como se puede comprobar en la Tabla 9 de frecuencias y porcentajes de dicha variable.

Tabla 9. En relación con la época anterior a la enfermedad sus relaciones con sus amigos, compañeros, etc. son

\begin{tabular}{|l|l|c|c|c|}
\hline & & Frecuencia & Porcentaje & Porcentaje acumulado \\
\hline \multirow{4}{*}{ Válidos } & peores & 2 & 5,1 & 5,1 \\
\cline { 2 - 5 } & iguales & $\mathbf{3 4}$ & $\mathbf{8 7 , 2}$ & $\mathbf{9 2 , 3}$ \\
\cline { 2 - 5 } & mejores & 3 & 7,7 & 100,0 \\
\cline { 2 - 5 } & Total & 39 & 100,0 & \\
\hline Perdidos & Sistema & 47 & & \\
\hline Total & & 86 & & \\
\hline
\end{tabular}

Como indica la Tabla 10, el estadístico Chi-Cuadrado correspondiente a la pregunta sobre sus relaciones con sus amigos, compañeros, etc., muestra cómo la prueba de Chi-Cuadrado presenta un valor de 50,923 con 2 grados de libertad y una significación asintótica de ,000. Indica que la relación entre las variables es significativa, es decir, la 
variable estar enfermo tiene relación de dependencia con la variable "en relación con la época anterior a la enfermedad sus relaciones con sus amigos, compañeros, etc. son...".

Tabla 10. Prueba de Chi-Cuadrado. En relación con la época anterior a la enfermedad sus relaciones con sus amigos, compañeros, etc. Son.

\begin{tabular}{|c|c|}
\hline & $\begin{array}{r}\text { En relación con la época anterior a la enfermedad sus relaciones } \\
\text { con sus amigos, compañeros, etc. son }\end{array}$ \\
Chi-cuadrado & $\mathbf{5 0 , 9 2 3}$ \\
\hline gl & 2 \\
Sig. asintót. &, 000 \\
\hline
\end{tabular}

6. A la pregunta “¿Y su situación laboral?”, efectuada a los 45 sujetos encuestados afectados por el Síndrome de Ardystil, contestaron 38 de ellos, de los cuales un 68,4\% manifiesta que su situación laboral "ha empeorado", un 21,1\% declaran que su situación laboral "sigue igual", frente a un 10,5\%, que manifiesta que dicha situación laboral "ha mejorado", como se muestra en la Tabla 11 de frecuencias y porcentajes y en el Gráfico 6, donde se indica la frecuencia de sujetos de la variable.

Tabla 11. ¿Y su situación laboral?

\begin{tabular}{|l|l|c|c|c|}
\hline & & Frecuencia & Porcentaje & Porcentaje acumulado \\
\hline \multirow{5}{*}{ Válidos } & ha empeorado & $\mathbf{2 6}$ & $\mathbf{6 8 , 4}$ & $\mathbf{6 8 , 4}$ \\
\cline { 2 - 5 } & sigue igual & 8 & 21,1 & 89,5 \\
\cline { 2 - 5 } & ha mejorado & 4 & 10,5 & 100,0 \\
\cline { 2 - 5 } & Total & 38 & 100,0 & \\
\hline Perdidos & Sistema & 48 & & \\
\hline Total & & 86 & & \\
\hline
\end{tabular}

La prueba de Chi-Cuadrado realizada a la variable “¿Y su situación laboral?" ofrece un valor de 21,684 con 2 grados de libertad y una significación asintótica de ,000, lo que indica que la relación entre las variables es significativa, es decir, la variable ¿Y sus situación laboral? está relacionada con la variable estar o no afectado por el Síndrome de Ardystil. Dichas variables son dependientes entre sí.

Tabla 12: Prueba de Chi-Cuadrado. ¿Y su situación laboral?

\begin{tabular}{|c|c|}
\hline & ¿Y su situación laboral? \\
\hline Chi-cuadrado & $\mathbf{2 1 , 6 8 4}$ \\
\hline gl & 2 \\
\hline Sig. asintót. &, 000 \\
\hline
\end{tabular}




\section{CONCLUSIONES Y DISCUSIÓN}

Se mantiene la satisfacción general acerca de las relaciones y apoyo tanto de los amigos como de la familia. El tiempo transcurrido desde la aparición de la enfermedad juega a favor del ánimo y el deseo de volver al trabajo. En muchos casos, está en la base de ese ligero incremento el síndrome depresivo, ya que encuentran que su situación y capacidad laboral han empeorado.

Los resultados encontrados a partir de los procedimientos estadísticos, en ningún caso deberían sustituir el análisis y seguimiento pormenorizado de cada caso individual. Se puede hablar de un "regreso a la normalidad", no excesivamente bien recibido por estos sujetos. Por lo tanto, podemos afirmar que al finalizar este estudio todas las hipótesis planteadas se confirman.

Esperamos que esta investigación pueda servir no sólo para poner de manifiesto desde un planteamiento investigador las secuelas de la enfermedad, sino también para que empresarios y trabajadores tomen conciencia de lo importante que es prevenir el riesgo laboral, la protección de la seguridad y salud en el trabajo, lo que hará posible una mejor calidad de vida (Cañedo, Guerrero, Salman, Cruz \& Pérez; 2006).

La prevención debe ser el objetivo más importante de la actividad empresarial, evitando o minimizando las causas de los accidentes y enfermedades profesionales. Se defiende un enfoque holista e integrado de la salud y la seguridad en las organizaciones que plantea la necesidad de vincular los conceptos, filosofías y estrategias de la cultura de seguridad y la cultura organizacional (Díaz, Isla, Rolo, Villegas \& Ramos; 2008).

Las condiciones de trabajo de las personas - principal valor de cualquier empresay su formación, constituyen un medio determinante para alcanzar niveles óptimos de calidad de productos, servicios y procesos, contribuyendo con ello a que la empresa sea eficiente y competitiva.

Únicamente con personas cualificadas y motivadas se puede lograr el nivel de innovación, creatividad y compromiso que las organizaciones necesitan. La prevención y en general la atención a las condiciones de trabajo, regida por principios éticos, es la mejor manera de lograr la confianza de los trabajadores, reforzar el liderazgo de los directivos y demostrar ante la sociedad el nivel de responsabilidad social asumido en materia laboral.

\section{BIBLIOGRAFÍA}

CAÑEDO, R., GUERRERO, J., SALMA, E., CRUZ, Y. y PÉREZ, G. (2006): “Calidad de vida y trabajo: algunas consideraciones útiles para el profesional de la información", Acimed, 14 (2).

DÍAZ, D., ISLA, R., ROLO, G., VILLEGAS, O., RAMOS, Y. y HERNÁNDEZ, E. (2008): "La salud y la seguridad organizacional desde una perspectiva integradora", Papeles del Psicólogo, 29 (1), 83-91. GIL, F. y ALCOVER DE LA HERA, C. (Coords.) (2003): Introducción a la Psicología de las Organizaciones, Madrid, Alianza.

IBARRA, E. (2000): "Teoría de la Organización, mapa conceptual de un territorio en disputa", en De La Yorza, E. (comp.). Tratado latinoamericano de sociología del trabajo. México, Fondo de Cultura Económica.

MARTÍN QUIROS, M. A. y ZARCO, V. (2009): Psicología del trabajo, de las organizaciones y de los Recursos Humanos, Madrid, Pirámide.

MAYO, G.E. (1933): The Humans Problems of an Industrial Civilization, New York, McMillan. 
MORENO, B. (2000): "Olvido y recuperación de los factores psicosociales en la salud laboral", Archivos de Prevención de Riesgos Laborales, 3 (1), 3-4.

OSCA, A. (2004): La Psicología de las Organizaciones, Madrid, Sanz y Torres.

PALACÍ, F. (coord.) (2005): Psicología de la Organización, Madrid, Pearson Prentice Hall.

PEIRÓ, J.M. y PRIETO, F. (1996): Tratado de Psicología del Trabajo (2 volúmenes), Madrid, Síntesis Psicología.

RODRÍGUEZ, A., DÍAZ, F., FUERTES, F., MARTÍN, M., MONTALBÁN, M.,

ROETHLISBERGER, J.F. y DICKINSON, W.J. (1939): Management and the Worker: an Account of a Research Program Conducted by the Western Electric Company, Hawthorne works, Chicago. Cambridge, Mass, Harvard Univ. Press.

ROMERAL HERNÁNDEZ, J. (2007): "El delegado de prevención: problemas prácticos”, Gestión práctica de riesgos laborales: Integración y desarrollo de la gestión de la prevención, 59, 24-29.

SÁNCHEZ, E. y ZARCO, V. (2004): Psicología de las Organizaciones, Barcelona, UOC.

\section{Breve currículo:}

\section{Adela Reig Botella}

Doctora por la Universidad de La Coruña. Experta en Seguridad y Salud Laboral. Ha escrito diferentes artículos relacionados con esta materia en diversas revistas especializadas, y asimismo ha participado en diferentes proyectos de investigación.

\section{Miguel Clemente Díaz}

Catedrático de Psicología Social en la Universidad de A Coruña. Es experto en Psicología Jurídica, materia en la que ha escrito libros (en editoriales como Pirámide, Biblioteca Nueva, EOS, Delta, y en esta última la obra Psicología Jurídica Laboral) y artículos (en revistas como Aggressive Behavior, The Journal of Social Psychology, Internatiuonal Journal of Psychology, así como en revistas españolas de prestigio). Ha desarrollado proyectos de investigación sobre esta temática. Ha sido Presidente de la Sección de Psicología Jurídica de los Colegios Oficiales de Psicólogos de Madrid y de Galicia. En la actualidad es el Director de la Unidad de Investigación en Psicología Jurídica y Protección del Menor de la Universidad de La Coruña. 